\title{
Intracavernous Pharmacotherapy for Management of Erectile Dysfunction in Spinal Cord Injury
}

\author{
L. K. Lloyd, MD, ${ }^{1}$ J. S. Richards, PhD $^{2}$ \\ ${ }^{1}$ Urological Rehabilitation and Research Center, Spain Rehabilitation Center, \\ ${ }^{2}$ Division of Urology, Department of Surgery and Department of Rehabilitation \\ Medicine, University of Alabama in Birmingham, Birmingham, Alabama 35294, \\ $U S A$
}

\section{Summary}

Sexual dysfunction due to inadequate or poorly sustained erections is a common problem among patients with spinal cord injury (SCI). While penile prostheses have provided adequate treatment for many patients, the complication rate in spinal cord injured patients remains high and they have not received widespread acceptance among SCI patients or the physicians treating them. Recently, intracavernous injections of vasoactive medications have proved beneficial for treating patients with erectile dysfunction. In the current study, we evaluated 40 SCI patients and 116 patients with vascular based impotence who had been followed for a minimum of 1 month while utilising intracavernous injections of phentolamine and papaverine. SCI patients with neurogenic based erectile dysfunction are significantly different from vascular impaired patients. SCI patients are younger and have been impotent for a longer period of time when treatment is sought. Furthermore, the dose of medication required is significantly less for SCI patients. The time of erection is longer and the quality of erection is better in neurogenic based impotence. Results with this treatment in SCI appear to be quite good with short term followup and long term evaluation of this methodology in SCI appears warranted.

Key words: Spinal cord injury; Sexual dysfunction; Papaverine; Intracavernous injection.

Sexual dysfunction in spinal cord injured (SCI) patients is a common problem and a significant aspect of the disability. Approximately 20 to $30 \%$ of patients with thoracic and cervical spine injuries have inadequate erections for successful coitus (Comarr, 1985). In patients with lower spinal cord or cauda equina injuries the outlook is less good. Most of these patients have only psychogenic erections and these are frequently unsustained or incomplete and therefore often less functional. Some of these patients, particularly those with incomplete 
injuries, are able to have successful coitus. There remains, however, a fairly large group of patients with injuries at all levels of the spinal cord who have sufficient erectile dysfunction to cause them to seek treatment. Fertility is a major problem among spinal cord injured males with the vast majority unable to ejaculate or father children.

With development of a variety of penile prostheses, the ability to treat erectile dysfunction was vastly improved (Scott, 1973; Small, 1975). This has resulted in new research into the mechanisms of erection and development of new treatment modalities. Penile implants have not received widespread acceptance among SCI patients or the physicians treating them in part because of the relatively high complication rate seen in these patients (Rossier, 1984; Golji, 1979). Psychosocial counselling is an important part in the rehabilitation process of SCI patients and many well rehabilitated patients rely upon other methods of sexual expression rather than penile-vaginal intercourse (Green, 1986). Recently, intracavernous pharmacotherapy has been utilised to treat a variety of patients with insufficient erections for coitus (Brindley, 1986; Zorgniotti, 1985; Sidi, 1986). Patients with neurological injury appear to respond quite well to pharmacological treatment and a few reports have appeared specifically related to SCI patients (Wyndaele, 1986; Beretta, 1986; Bodner, 1987; Sidi, 1987). We began a programme utilising papaverine, a smooth muscle relaxant and phentolamine, an alpha adrenergic blocker, in August 1985. Patients with a variety of aetiologies producing sexual dysfunction have been treated. This report focuses on the response in SCI patients and highlights differences in response and complication rates between vascular based and neurologically based impotence.

\section{Materials and methods}

In August 1985 we began a clinical programme for the treatment of male erectile dysfunction with intracavernous phentolamine and papaverine. Since these agents were not, and are still not, FDA-approved for this purpose, we sought and received Institutional Review Board approval for our evaluation and treatment protocol. All patients entering into the treatment protocol were required to sign a detailed consent form explaining the risks and benefits of the treatment programme. Patients presenting to the general urology clinic underwent history and physical examination, determination of serum testosterone and prolactin levels, and snap gauge nocturnal penile tumescence monitoring. Selected patients underwent additional testing including urodynamic evaluation, bulbocavernosus latency testing, paraspinal electromyography, and nerve conduction velocities in the lower extremities. A few patients underwent formal nocturnal penile tumescence monitoring in the sleep laboratory. Doppler-assisted penile brachial index was performed only in select patients. Patients with obvious neurological disease such as spinal cord injury, did not undergo nocturnal tumescence studies or serum hormone determinations, except in those with very incomplete injuries. Patients with spinal cord injuries did undergo extensive neurological testing to determine the level and completeness of injury in addition to psychological evaluation and counselling with particular reference to sexual alternatives and realistic expectations prior to beginning the treatment programme. 
After completing the above preliminary evaluations, patients were classified into the following groups: (1) vascular; (2) diabetes mellitus; (3) psychogenic; (4) neurogenic; (5) mixed; and (6) undetermined. At the time of the initial office visit patients were counselled briefly regarding the pharmacological treatment protocol and given literature to read at home regarding the injection programme as well as the detailed consent form. At the return office visit, results of testing were discussed and treatment alternatives discussed in some detail. Patients who elected to participate in the intracavernous injection protocol were given a test injection in the office with monitoring of blood pressure and erectile response for approximately 30 minutes post-injection. Those who appeared to have sufficient response to the medication were instructed in the injection technique and given a trial period of 1 month at home for determination of clinical response. The wife or partner of patients such as quadriplegics, who had insufficient dexterity, was taught to give the injections. SCI patients were instructed to void, remove the condom catheter if one was worn, and to cleanse the genitalia carefully with soap and water prior to the administration of an injection.

Initially we used a mixture of $30 \mathrm{mg} / \mathrm{ml}$ papaverine hydrochloride and 0.5 $\mathrm{mg} / \mathrm{ml}$ of phentolamine mesylate. We administered $1 \mathrm{ml}$ of this mixture to patients with vasculogenic impotence and $0.5 \mathrm{ml}$ (15 mg papaverine and 0.25 mg of phentolamine) to patients with neurogenic, psychogenic and mixed aetiologies. Patients used a $1 \mathrm{ml}$ insulin syringe with a 28 gauge needle and injected unilaterally into the corpus cavernosum. They were instructed to alternate sides and vary the injection site along the lateral aspect of the penis. Manual compression of the injection site was applied for 1 minute post-injection. Dose was adjusted upward or downward, depending upon both initial response with the test injection and clinical response during the 1 month trial period.

At follow-up, response was assessed according to the frequency of use, length of time erections lasted, and quality of erection rated subjectively by the patient on a 10-point scale. Patients were examined by careful palpation for any evidence of corporeal fibrosis and for any other possible complications related to the treatment protocol. All data was entered into an office based computer system and updated periodically with follow-up. Differences in the vascular and SCI groups were assessed for statistical significance using Student's twotailed t-test.

\section{Results}

Two hundred and twenty eight patients were entered into the protocol between August 1985 and April 1988. There were 41 SCI patients $\left(18^{\circ}{ }_{0}\right)$. One hundred and eighty three patients had a 1-month minimum follow-up. Twenty seven of these patients had psychogenic based impotence and were excluded from the current report. Included in the current report are 156 patients: 116 with vascular based impotence and 40 SCI patients. In the SCI group, there were 32 paraplegics, 10 incomplete, and 8 quadriplegics, 4 incomplete. Follow-up, age and length of time impotent are shown in Table I. Length of follow-up was significantly longer in SCI patients with a mean of 11.8 months. SCI patients were younger than vascular patients and the length of time impotent was longer. A comparison of the response between vascular and SCI patients is shown in 
Table I Comparison of patient data in vascular and SCI groups

\begin{tabular}{lccc}
\hline & Vascular & SCI & Total \\
\hline Patients & 116 & 40 & 156 \\
Follow-up (months) & $4 \cdot 9$ & $11 \cdot 8$ & $6 \cdot 6$ \\
Age (years) & $54 \cdot 8$ & $34 \cdot 7$ & $49 \cdot 6$ \\
Time impotent (years) & $2 \cdot 8$ & $5 \cdot 8$ & $3 \cdot 5$ \\
\hline
\end{tabular}

All differences significant with $\mathrm{P}<.001$

Table II Comparison of response rates in vascular and SCI impotence

\begin{tabular}{llll}
\hline & Vascular & SCI & Total \\
\hline Dose (ml) & $1 \cdot 15$ & 0.5 & 0.98 \\
Frequency use (n/week) & 1.4 & 0.9 & 1.3 \\
Time of erections (hours) & 1.26 & 1.96 & 1.45 \\
Quality erections (N/10) & 7.5 & 8.7 & 7.8 \\
\hline
\end{tabular}

All differences significant with $\mathrm{P}<\cdot 01$

Table II. Patients with vascular based impotence required a higher dose of medication and used it more frequently than did SCI patients. Erections among SCI patients lasted longer and the quality of erections was significantly better than in vascular patients. About one quarter of the patients discontinued treatment for a variety of reasons and there was no significant difference among percentage of SCI patients compared to percentage of vascular based patients who discontinued treatment. Reasons for discontinuing treatment in the 2 groups of patients are shown in Table III. As can be seen, the predominant reason for vascular patients to discontinue the programme was poor erection quality. Many of these proceeded on to other forms of therapy. Only 1 SCI patient discontinued the programme because of an insufficient response and he declined additional evaluation or treatment.

Complications occurred frequently in both groups of patients (Table IV). Twenty one $(52 \%)$ of SCI patients experienced some complication with the injection protocol while only $21 \%$ of the vascular patients experienced a complication. Fortunately, most complications were minor and relatively easily managed. As can be seen, priapism and prolonged erection were seen much more commonly in the SCI group as was ecchymosis. Prolonged erection was defined as a full erection lasting greater than 4 hours and priapism defined as a full erection lasting greater than 6 hours or producing pain. Corporeal fibrosis was seen more commonly in vascular patients, even though the follow-up in the SCI group was longer. The increased incidence of priapism and prolonged erection observed during the course of the study led us to revise the dosage recommendations and more recently, SCI patients have been started on an initial dose of $0.3 \mathrm{ml}(10 \mathrm{mg})$ of papaverine only. While priapism was seen in only 2 patients in the current series it was seen overall in 7 of 228 patients $(3 \%)$ who received initial injections in the office. In addition to the 2 SCI patients, there were 2 patients with psychogenic impotence, 1 with neurogenic impotence, 1 mixed and 1 vascular who was probably misdiagnosed. Interestingly, the 2 SCI patients did not have priapism on the initial test injection but it occurred after several months when each patient arbitrarily increased his dose. 
Table III Reasons for discontinuing treatment

\begin{tabular}{lcl}
\hline & Vascular & SCI \\
\hline Poor erections & 15 & 1 \\
Did not like & 6 & 2 \\
Erections returned & 3 & - \\
Painful & 2 & - \\
No partner & - & 3 \\
Could not afford & 1 & 1 \\
Other & 5 & 2 \\
Total & 32 & 9 \\
\hline
\end{tabular}

Table IV Complications during follow-up

\begin{tabular}{llll}
\hline & Vascular & SCL & Total \\
\hline & $\mathrm{N}=116$ & $\mathrm{~N}=40$ & $\mathrm{~N}=156$ \\
Priapism & $0(0)$ & $2(5)$ & $2(1 \cdot 2)$ \\
Prolonged erection & $5(4 \cdot 3)$ & $7(17)$ & $12(7 \cdot 6)$ \\
Ecchymosis & $9(7 \cdot 7)$ & $8(20)$ & $17(11)$ \\
Fibrosis & $7(6)$ & $1(2 \cdot 5)$ & $88(5)$ \\
\hline
\end{tabular}

Management of priapism has been accomplished easily in all of our patients by simply irrigating the corpora with phenylephrine $0.02 \mathrm{mg}$ per $\mathrm{ml}$. No patient has required a surgical shunting procedure. Autonomic dysreflexia occurred only once on an initial injection. It was mild and did not recur with subsequent injections.

Two complications occurred in SCI patients that could not be clearly related to the treatment protocol other than temporally. One patient developed suprapubic pain and altered bladder function with urinary retention shortly after beginning the intracavernous programme. This did not appear to be urinary retention related to prolonged erection and the exact aetiology of this apparent neurological alteration was not determined. It gradually resolved over time and the patient has elected not to resume the programme to date. Another patient had the onset of a seizure disorder after being on the protocol for several months and evaluation has not disclosed a specific aetiology for the seizures. The seizures have continued even after he has stopped the treatment protocol and he is presently under continuing evaluation by the neurologist.

Overall, the majority of patients were quite satisfied with the programme and felt that it opened a new option for treatment. Thus far we have not observed declining response among the SCI patients, although this is certainly a concern during long term follow-up in this relatively young group of patients.

\section{Discussion}

Virag in 1982 and Brindley in 1983 introduced the concept of intracavernous injection of vasoactive drugs to produce a functional erection. Papaverine is a smooth muscle relaxant which produces both vasodilation and relaxation of the sinusoidal spaces in the corpus cavernosum. Phentolamine is a short-lived alpha-adrenergic blocking agent that produces vasodilation. The combination of 
increased arterial inflow as a result of vasodilation and engorgement of the relaxed sinusoidal spaces causes compression of the subalbugineal venous plexus resulting in decreased outflow of blood and an erection (Lue, 1987). Patients with all types of erectile dysfunction have been shown to respond to the intracavernous injection of these agents (Zorgniotti, 1985; Lue, 1987). Patients with vascular disease appear to respond less well and it has been suggested that the differential response to these agents can also function as a diagnostic test (Virag, 1984). Zorgnoitti and Lefleur introduced the use of intracavernous injection in this country in 1985 and since that time, a number of reports have appeared attesting to the efficacy of these agents. The reports have been generally laced with cautious optimism since long term effects are unknown and since these agents have not been approved by the FDA for use in this manner.

Several reports have appeared describing the results of treatment in men with spinal cord injury (Wyndaele, 1986; Beretta, 1986; Bodner, 1987; Sidi, 1987). SCI males are in general a younger group of patients and hence more likely to have more normal vasculature. These patients with neurogenic based impotence appear to be exquisitely sensitive to the intracavernous agents and hence a lower dose of medication is required. In the current series, the dose for SCI patients was significantly lower than for vascular based impotence. The time of erections was longer and the quality of erections was significantly better in the SCI group. This better response in neurogenic based impotence does place these patients at greater risk for prolonged erection and priapism. Our initial experiences using a combination of phentolamine and papaverine in SCI patients did, in fact, result in these problems in a small number of patients. Since we revised our dosage schedule to start the injection programme with a dose of $0.3 \mathrm{ml}(10 \mathrm{mg})$ papaverine, we have not had any additional cases of priapism or significant problems with prolonged erection. Other investigators have likewise advised an initial dose of papaverine in a similar dosage range to avert these potential complications (Bodner, 1987; Sidi, 1987). It is then quite easy to adjust the dose upward as needed should the response to the initial dose be inadequate. We have permitted patients to incrementally increase the dose by $0.1 \mathrm{ml}$ if the initial dosage proved inadequate after the first few injections, but we have advised them not to carry out an injection more than once in a 24-hour period.

There are several problems of potential concern with the use of intracavernous injections in SCI patients. Patients with spinal cord lesions above T5 may be prone to episodes of autonomic dysreflexia. We saw one such case in the current series with the initial test injection. It was mild and transient, however, and did not require treatment. This patient has subsequently utilised selfadministered injections on numerous occasions without autonomic dysreflexia. Bodner and associates in their review of 20 patients found no incidence of autonomic dysreflexia, nor has it been reported in other series dealing with SCI patients.

Chronic or recurrent urinary tract infection is a problem in SCI patients regardless of the method of bladder management. Montgomerie and Morrow (1978) have shown that significant colonisation of the perineal and genital skin with pathogenic gram negative organisms occurs in a high percentage of SCI 
patients. This coupled with the use of condom catheters in the majority of our male patients gave us concern that cellulitis or cavernositis might develop as a result of the injections. All patients were cautioned to empty the bladder, remove the condom and cleanse the genitalia carefully with soap and water. As a result we have not seen any cases of cavernositis or cellulitis to date. No other major series dealing with SCI patients has reported cavernositis or cellulitis but 2 cases from Japan were cited in Lue and Tanagho's review of pharmacotherapy. All patients entering a self-injection programme should be carefully monitored by a qualified urologist with reinforcement of proper techniques at follow-up visits.

Fibrosis of the corpus cavernosum has been reported with experimental papaverine injections in monkeys (Abozeid, 1987). There have been several case reports of fibrosis occurring in humans after varying periods of utilisation of intracavernous injections of both phentolamine and papaverine (Larsen, 1987; $\mathrm{Hu}, 1987$ ). Additionally, most of the major series have mentioned small numbers of cases where fibrosis was thought to occur after long term use of the injections. Bodner et al. reported 1 patient out of 20 who developed a firm area at the injection site but they suspected that it was related to subcutaneous injection. Sidi et al. reported 1 case of localised intracorporeal induration in a spinal cord patient after 11 months use of the injection once a week. In the current series, fibrosis appeared to occur less often in SCI patients than vascular based impotence, even though the follow-up with SCI patients was over twice as long as with vascular patients. This may be related to the less frequent use seen in spinal cord patients or the much lower doses required for functional erection. Brindley recommends limiting the use of injections to twice a month in hopes of minimising the risks of such complications (Brindley, 1986). The exact aetiology of the fibrosis is unclear but it seems likely that it will occur in at least a small percentage of patients and that its incidence may increase over time. Clearly, additional long term follow-up is needed to assess both the incidence of corporeal fibrosis and its effect on the continuation of the injection programme as well as its effect on subsequent therapies.

Overall, the patient response to the injection programme has been extremely positive. Patients, as well as their partners, derive enormous psychological benefit from restoration of erectile function that permits penile-vaginal intercourse (Althof, 1987). Psychological counselling both before and during treatment remains an important part of the long term management, particularly in this select group of patients (Green, 1986). This medical form of managing erectile dysfunction appears to have attracted relatively large numbers of patients who, at least initially, would not elect a surgical option. Penile prostheses have been a reliable method of treating organic erectile dysfunction but as noted previously the complication rate in SCI is quite high due primarily to the risks of erosion and infection (Rossier, 1984; Golji, 1979). For this reason, intracavernous pharmacotherapy is certainly an attractive and highly successful method of managing the erectile dysfunction of SCI. Mean follow-up in the current series, however, is only 12 months, only slightly longer than other reported series in SCI. It must be emphasised that careful supervision and long term follow-up of these patients is manda- 
tory to fully assess both the benefits and risks of long term intracavernous therapy.

\section{References}

Abozeid M, JUenemann KP, Luo J et al. 1987 Chronic papaverine treatment: the effect of repeated injections on the simian erectile response and penile tissue. Fournal of Urology 138:1263-1266.

Althof SE, TURNer LA, Levine SB et al. 1987 Intracavernosal injection in the treatment of impotence; a prospective study of sexual, psychological, and marital functioning. Fournal of Sex and Marital Therapy 13:155-167.

Beretta G, Zanollo A, Fanciullacci F, et al. 1986 Intracavernous injection of papaverine in paraplegic males. Acta Europaea Fertilitatis 17:283-284.

BODNER DR, LINDAN R, LEFFLER E et al. 1987 The application of intracavernous injection of vasoactive medications for erection in men with spinal cord injury. Fournal of Urology 138:310-311.

BRINDLEY GS 1983 Cavernosal alpha-blockade: a new technique for investigating and treating erectile impotence. British fournal of Psychiatry 143:332-337.

BRINDLEY GS 1986 Maintenance treatment of erectile impotence by cavernosal unstriated muscle relaxant injection. British fournal of Psychiatry 149:210-215.

COMARR AE 1985 Sexuality and fertility among spinal cord and/or cauda equina injuries. Fournal of the American Paraplegia Society 8:67-75.

GolJI H 1979 Experience with penile prosthesis in spinal cord injury patients. Fournal of Urology 121:288-289.

GREEN BG, SLOAN SL 1986 Penile prostheses in spinal cord injured patients: combined psychosexual counseling and surgical regimen. Paraplegia 24:167-172.

Hu KN, Burks C, ChrISTY WC 1987 Fibrosis of tunica albuginea: complication of long-term intracavernous pharmacological self-injection. Fournal of Urology 138:404-405.

LARSEN EH, GASSER TC, BRUSKEWITZ RC 1987 Fibrosis of corpus cavernosum after intracavernous injection of phentolamine/papaverine. Fournal of Urology 137:292-293.

LUE TF, TANAGHO EA 1987 Physiology of erection and pharmacological management of impotence. Fournal of Urology 137:829-836.

Montgomerie JZ, MORROw JW 1978 Pseudomonas colonization in patients with SCI. Fournal of Epidemiology 108:328-336.

ROSSIER AB, FAM BA 1984 Indications and results of semi-rigid penile prostheses in spinal cord injury patients: longterm followup. Fournal of Urology 131: 59-61.

Scott FB, BRADley WE, TIM GW 1973 Management of erectile impotence. Use of implantable, inflatable prostheses. Urology 2:80-82.

Sidi AA, CAMERON JS, DUfFy LM et al. 1986 Intracavernous drug induced erections in the management of male erectile dysfunction: experience with 100 patients. Fournal of Urology 135:704-706.

Sidi AA, CAmeron JS, Dykstra D et al. 1987 Vasoactive intracavernous pharmacotherapy for the treatment of erectile impotence in men with spinal cord injury. Fournal of Urology 138:539-542.

SMALL MP, CARRION HM, GoRDON JA 1975 Small-Carrion penile prosthesis. Urology 5:479486.

VIRAG R 1982 Intracavernous injection of papaverine for erectile failure. The Lancet 2:938.

VIRAG R, FRYDMAN D, LEGMAN M et al. 1984 Intracavernous injection of papaverine as a diagnostic and therapeutic method in erectile failure. Angiology 35:79-82.

WYNDAELE JJ, DE MEYER JM, DE SY WA et al. 1986 Intracavernous injection of vasoactive drugs, an alternative for treating impotence in spinal cord injury patients. Paraplegia 24:271-275.

ZORGNIOTTI AW, LeFLEUR RS 1985. Auto injection of the corpus cavernosum with a vasoactive drug combination for vasculogenic impotence. Fournal of Urology 133:39-41. 